\title{
Características de utilização de Software as a Service: Um estudo sobre as operadoras de planos de saúde
}

\author{
Marcus Sucupira, Fábio Correa, Luiz Cláudio Gomes Maia, Ana Maria Pereira \\ Cardoso, Fernando Silva Parreiras \\ Laboratório de Sistemas de Informação Avançados (LAIS), Universidade FUMEC \\ Av. Afonso Pena, 3880 - 30130-009 \\ Belo Horizonte, MG, Brasil \\ mvsucupira@fumec.edu.br, fabiocontact@gmail.com, \\ \{luiz.maia, ana.cardoso, , fernando.parreiras\}@fumec.br
}

\begin{abstract}
The current scenario of Health Insurance Providers (HIP) consists of a steady increase in operating expenses over revenues, growing at a slower pace. Among several factors that explain this scenario, this work was highlighted two important points, they are: (i) The requirements of regulation imposed on private health care sector with the creation of the ANS in 2000; and (ii) the increased use and costs of physician - hospital procedures resulting from population aging. The objective of this work was a study of the market for Health Insurance Providers (HIP) to analyze the motivators and inhibitors of using systems in SaaS format.Considering the context presented, it is believed that the rational use of information systems, especially with the paradigm of cloud computing, and more specifically with the adoption of SaaS (type of software available in the cloud) could be a way for providers to optimize their health plan budgets technology. The methodology used in this work was the application of a survey to analyze the factors influencing adoption of SaaS technology, based on technology acceptance model (TAM). In this context, expected the influence of factors on the use of SaaS technology in organizations, and through the application of the survey, the motivating factors and inhibitors of technology adoption SaaS in Health Plans Operators market.
\end{abstract}

\begin{abstract}
O atual cenário das empresas operadoras de planos de saúde (OPS) consiste em um aumento nas despesas operacionais em detrimento das receitas, que crescem em um ritmo lento. Este fato pode ser justificado por dois fatores principais: (i) As exigências da regulação impostas ao setor de saúde suplementar a partir da criação da ANS no ano 2000; e (ii) Aumento da utilização e do custo dos procedimentos médico-hospitalares decorrentes do envelhecimento populacional. Como problema de pesquisa, foi definido a análise
\end{abstract}

dos fatores motivadores e inibidores de uso de sistemas no modelo SaaS em operadoras de planos de saúde. A metodologia deste projeto de pesquisa foi a realização de um survey, com base no modelo de aceitação de tecnologia TAM ( $T e-$ chnology Acceptance Model). Considerando esse contexto, acredita-se que o uso racional de sistemas de informação, principalmente com o paradigma da computação em nuvem, especificamente com a adoção de SaaS (Software as a Service), pode ser um caminho para as operadoras de plano de saúde otimizarem os seus orçamentos em tecnologia.

\section{INTRODUÇÃO}

A área da saúde é um campo de estudo sensível em relação às mudanças tecnológicas. Desta forma, estudos e pesquisas de tecnologia aplicada à área da saúde enfrentam o desafio de atualizar dados sobre a adoção de tecnologia no setor.

A aparição do fenômeno da computação em nuvem representa uma mudança fundamental na maneira pela qual os serviços de tecnologia da informação são inventados, desenvolvidos, implantados, escalados, mantidos e remunerados [20]. De acordo com [16] a computação como a conhecemos hoje é um paradoxo, de um lado, os computadores estão se tornando mais potentes com o passar do tempo, ao mesmo tempo em que o seu custo decresce rapidamente, transformando-se em commodity. Por outro lado, a computação está cada vez mais entranhada nas organizações, e o aumento da complexidade no gerenciamento de toda a infraestrutura está fazendo com que o seu custo aumente [25].

Este trabalho teve como objetivo apresentar os fatores de influência no uso de softwares que utilizam este paradigma de computação em nuvem nas operadoras de planos de saúde. Foi proposto responder a seguinte pergunta de pesquisa: Quais são os fatores motivadores e inibidores na adoção de Software as a Service (SaaS) na empresas operadoras de planos de saúde no Brasil?

[20] afirma que o ímpeto atual pela mudança do paradigma computacional ainda é predominamente pela perspectiva de custos, uma vez que as organizações estão descobrindo que os substanciais investimentos em tecnologia da informação são frequentemente subutilizados. Esta afirmação pode ser constatada por uma pesquisa conduzida no Gartner Research [24], mostrando que aproximadamente dois terços do orçamento das equipes de TI das empresas são voltadas para 
as rotinas de suporte e manutenção.

O atual cenário das empresas operadoras de planos de saúde representa um constante aumento nas despesas operacionais em detrimento das receitas, que crescem em um ritmo mais lento. De acordo com a [1], as despesas operacionais das operadoras de saúde aumentaram a uma taxa de 92,3\%, passando, de 41,7, para 80,2 bilhões de reais no período de 2007 a 2012. No mesmo período, a receita das operadoras aumentaram $82,7 \%$, passando de 52,2 bilhões de reais, em 2007, para 95,4 bilhões de reais no encerramento do ano de 2012.

A relevância do assunto está dois fatores: (i) as exigências da regulação impostas ao setor de saúde suplementar a partir da criação da ANS no ano 2000; e (ii) ao aumento da utilização e do custo dos procedimentos médico-hospitalares decorrentes do envelhecimento populacional.

Os avanços na disponibilização de soluções de computação em nuvem vêm sendo vistos como uma nova possibilidade para a redução nos volumes de investimento em tecnologia da informação, habilitando maior flexibilidade na demanda por serviços, com custos atrativos [3]. Dessa maneira, acredita-se que o uso racional de sistemas de informação, principalmente com o paradigma da computação em nuvem, e mais especificamente com a adoção de SaaS, pode ser um caminho para as operadoras de plano de saúde otimizarem os seus orçamentos em termos de gastos com tecnologia.

A computação em nuvem representa a convergência de duas tendências em tecnologias da informação: (a) eficiência em TI, por meio do poder de computadores sendo utilizados eficientemente, utilizando-se do rápido escalonamento no uso dos recursos de hardware e software; e (b) agilidade no negócio, por meio do uso da TI como uma ferramenta competitiva, como desenvolvimento ágil, processamento paralelo de dados, uso intensivo de business intelligence e aplicações móveis que respondem em tempo real aos requisitos dos usuários [15].

Este artigo está estruturado em sete partes. A primeira delas tratou da introdução. Na segunda são apresentados os fundamentos para o trabalho. A terceira apresenta trabalhos relacionados. A seção quatro mostra a metodologia utilizada para a coleta de dados. A quinta relata a análise realizada sobre os dados, sendo que os resultados são tratados na seção seis e, finalmente, a seção sete apresenta as conclusões decorrentes do estudo realizado.

\section{TRABALHOS RELACIONADOS}

Durante a uma revisão de literatura, não foram encontrados trabalhos relacionados à adoção de tecnologia SaaS em operadoras de planos de saúde. Foram utilizadas as seguintes fontes de busca: ScienceDirect, Cambridge Journal Online, ACM DL, Springer Link, IEEE Xplore, Wiley Online Library e Emerald Insight. Trabalhos internacionais relacionados ao tema foram levantados, porém nenhum deles apresentou resultados aplicáveis à situação em estudo.

Os trabalhos possuem natureza variada, em termos de propósito de pesquisa, ponto de vista de avaliação (clientes, fornecedores ou ambos) e, em alguns casos, aplicação de softwa- res específicos. Foram identificados sete trabalhos que utilizaram a teoria Techonology Percept and Model(TAM) como base para a construção de modelos e pesquisas.

[31] propõe um modelo exploratório para examinar os fatores importantes que afetam na adoção de SaaS como o objetivo de facilitar o entendimento de adoção de soluções SaaS. Além do uso do modelo TAM, o autor utiliza outras teorias adicionais, como o esforço de marketing, segurança e confiança. O propósito deste trabalho é dar subsídios tanto para utilizadores das tecnologias, quanto para os fornecedores, que podem utilizar essa pesquisa como fonte de informações para o desenvolvimento de produtos e mercados. Em seu outro trabalho, [32] aborda especificamente os fatores que influenciam a adoção de SaaS por usuários corporativos. Ele utiliza Rough Set Theory (RST), em conjunto com TAM. Um estudo empírico com empresas em Taiwan foi realizado e gerou uma séries de informações importantes sobre as necessidades dos usuários nas empresas no que tange ao uso de SaaS.

O trabalho de [12] é voltado para o estudo da aprendizagem de utilização de produtos, em momentos de experimentação (tryout) de tecnologias pelos usuários. Esse tipo de venda é comum na oferta de SaaS, e é um momento importante e decisivo no processo de aquisição da tecnologia. A proposta da pesquisa é um desenvolvimento de um modelo baseado nas teorias de aceitação de produto, modelos de ciclo de vida do consumidor, usabilidade e pesquisas em aceitação de tecnologias.

[7] desenvolveram e testaram um modelo de aceitação chamado SaaSQual, com base no conceito de qualidade de serviços digitais (e-services). Esse conceito é essencialmente dividido em quatro dimensões: facilidade de uso, segurança, confiabilidade e responsividade, que são oriundos da TAM. Além desses construtos, a influência social foi incluída como um componente importante de avaliação sobre a intenção no uso de sistemas.

[18] em seu trabalho, realizaram um estudo específico de adoção de sistemas ERP (Enterprise Resource Planning) na modalidade SaaS para pequenas e médias empresas. O foco do estudo foi identificar e avaliar os problemas comuns na fase de implementação e pós-implementação de ERPs. Foi realizada uma pesquisa qualitativa em cinco empresas européias e norte-americanas, que implementaram tais sistemas na modalidade SaaS.

[9] procuram saber sobre os fatores que afetam a aceitação, em pequenas e médias empresas, de tecnologias para desenvolvimento de websites na modalidade SaaS. São analisados os fatores externos que influenciam na aceitação deste modelo de fornecimento de tecnologia e se baseia no modelo TAM e da teoria de confirmação de expectativa. [30] propuseram um modelo teórico para investigar os efeitos do marketing estimulado, na forma de degustação e preço, no processo de decisão de aceitação de tecnologia. Foi realizado uma pesquisa utilizando uma plataforma móvel de fornecimento de jornal em uma amostra de 192 respondentes.

A computação em nuvem na modalidade SaaS permite perspectivas de análise em diversos campos conforme relatado e 
explorado nas pesquisas encontradas. Este artigo apresenta resultado de sua adoção e aceitação nas Operadoras de Planos de Saúde.

\section{METODOLOGIA}

Como técnica de coleta de dados, foi usado o levantamento tipo survey, que usa um instrumento único, com o uso de técnicas de amostragem, análise e inferência estatística [22]. A pesquisa com survey pode ser referida como sendo a obtenção de dados ou informações sobre as características ou as opiniões de determinado grupo de pessoas, indicado como representante de uma população-alvo, utilizando um questionário como instrumento de pesquisa $[4,8]$.

Levantamento do tipo survey também são chamados de pesquisa de avaliação por avaliar uma amostra representativa da situação problemática a ser investigada com objetivo de extrair conclusões acerca dessa amostra [22]. [8] ainda sugerem a classificação da survey quanto ao seu propósito e momento. Esta pesquisa tem o propósito descritivo pois visa identificar quais situações, atitudes, opiniões serão manifestados por uma determinada população com relação as características da adoção de SaaS pelas empresas, e é de natureza exploratória, pois objetiva entender um tema ainda pouco conhecido [2]. Quanto ao momento, a pesquisa será interseccional, que, para [2] são dados colhidos em um certo momento de uma amostra selecionada para descrever alguma população maior na mesma ocasião.

Após revisão da literatura, um questionário foi escolhido, baseado no trabalho de [7], que tem como suporte teórico o modelo escolhido, TAM. [22] ressalta que o pesquisador não interfere ou pouco interfere nas variáveis de pesquisa, pois são oferecidas pela natureza ou derivadas de uma teoria. As variáveis de pesquisa identificadas são avaliadas pelos respondentes do questionário, que é o instrumento de coleta de dados para a pesquisa. Em cada questão é apresentado uma afirmação e solicitado ao respondente que dê sua resposta usando uma escala de classificação. Para [11] a medição envolve a atribuição de números para uma variável e esses números devem refletir as características do fenômeno que está sendo estudado, o que é possível através do uso de escalas.

TAM tem sido amplamente utilizado para a investigação de como os usuários adotam uma tecnologia [31]. [5] propôs a versão original da TAM, baseado na Theory of Reasoned Action, com o propósito de testar como os usuários vêm a aceitar e a usar sistemas de informação. [17] indicaram que a TAM habilita uma organização a identificar os efeitos de variáveis externas referentes à relação causal entre a Percepção de Utilidade (PU), Percepção da Facilidade de Uso (PFU) e a Intenção Comportamental (IC). Dessa maneira, ajuda as organizações com a implementação e aplicação de sistemas de tecnologia. No que diz respeito à versão original da TAM, [5] argumenta que (1) a motivação do usuário pode ser explicada por três fatores: PU, PFU e Atitude Para Usar (APU); (2) a APU é o maior determinante de que o usuário realmente vai utilizar o sistema; (3) a APU é afetada por duas crenças: PU e PFU; (4) PU é diretamente influenciada pela PFU; e (5) essas duas crenças são diretamente influenciadas pelas características de design do sistema. Subsequentemente, o modelo original da TAM foi sendo modificado, como a primeira versão modificada [6], a última versão modificada [27], TAM2 [28], a Unified Theory of Acceptance an Use of Technology (UTAUT) [29], e TAM3 $[26]$

Optou-se pela utilização da adaptação do TAM aplicada no trabalho de [7] em que se aborda a elaboração de um modelo de avaliação dos fatores de influência na utilização de sistema no modelo SaaS, com base nos conceitos de qualidade em serviços digitais (e-services). O modelo proposto, denominado SaaSQual, avalia as relações diretas e indiretas na intenção de usar o SaaS com base nos parâmetros de qualidade de serviços digitais, da influência social e da percepção de utilidade. Sendo que esse último também é influenciado pelos elementos citados anteriormente como pode ser visto na figura 1. Após a escolha do questionário, foi realizado um trabalho de tradução, uma vez que as perguntas originais estão na língua inglesa. A tradução do questionário foi realizado pelo próprio autor da pesquisa. Uma vez acertado o questionário, ele foi aplicado a um grupo selecionado pela conveniência da facilidade de acesso, como forma de pré-teste. O objetivo foi validar o instrumento e determinar os ajustes necessários. Nesta pesquisa as respostas foram dadas em uma escala Likert, que, de acordo com [2], mostra-se aos respondentes uma afirmação e procura-se medir o grau de concordância ou aderência a ela por meio de uma escala que vai da total aceitação à total rejeição. Para esta pesquisa, foi adotado a escala de números de um (1) a sete (7), onde o número um representa a concordância total enquanto o número sete representa a discordância total. O número quatro é uma posição neutra do respondente.

\subsection{População}

Para esta pesquisa, optou-se por aplicar o questionário a toda a população pesquisada, que no caso são todas as operadoras de plano de saúde com registro ativo na ANS. A escolha pela aplicação ampla do questionário é justificado por: (i) facilidade de obtenção dos dados de contatos de todas as operadoras, uma vez que todas as informações de contato estão disponibilizadas no sítio oficial da ANS e são de domínio público; e (ii) pelo risco em não obter as respostas necessárias em uma amostra determinada, e, portanto quanto mais amplo for a aplicação do questionário, melhores serão as chances de obtenção de um maior número de respostas.

Após o questionário validado e ajustado, ele foi publicado oficialmente como survey eletrônico para obter respostas de empresas operadoras de plano de saúde das diferentes regiões do Brasil. A coleta dos dados é uma fase importante na investigação, para [22] o pesquisador tem pouco controle sobre ela, porém pode tomar algumas ações para aumentar a taxa de retorno. Como uma dessas ações, no momento do lançamento da survey, buscou-se o apoio para divulgação junto às entidades associativas da categoria, sendo elas a SBIS (Sociedade Brasileira de Informática na Saúde) e as Centrais Regionais da Unimeds do Brasil. O survey foi lançado no dia $27 / 08 / 2014$ e ficou no ar durante quatro meses, sendo encerrado no dia 23/12/2014. Ao final do dia 22 de dezembro de 2014 o questionário foi encerrado com um total de 174 respostas, sendo dessas 147 respostas válidas.

Os dados obtidos pelo survey foram analisados estatistica- 
mente com recurso a técnicas descritivas, análise fatorial, análise de correlação, Alfa de cronbach, análise de conglomerados, teste $t$ de student para amostras independentes $\mathrm{e}$ teste do qui-quadrado.

\subsection{Construtos aplicados à pesquisa}

$\mathrm{O}$ modelo utilizado nesta pesquisa, é o mesmo aplicado no trabalho de [7] onde abordam a elaboração de um modelo de avaliação dos fatores de influência na utilização de sistema no modelo SaaS, com base nos conceitos de qualidade em serviços digitais (e-services). O modelo proposto, denominado SaaSQual, avalia as relações diretas e indiretas na intenção de usar o SaaS com base nos parâmetros de qualidade de serviços digitais, da influência social e da percepção de utilidade. Sendo que esse último também é influenciado pelos elementos citados anteriormente, ver figura 1.

O modelo SaaSQual foi criado considerando quatro variáveis que foram testadas e refinadas, conforme tabela 1. De forma complementar, foram incluídos, também por [7], mais três variáveis que já haviam sido avaliadas e testadas, conforme tabela 2.

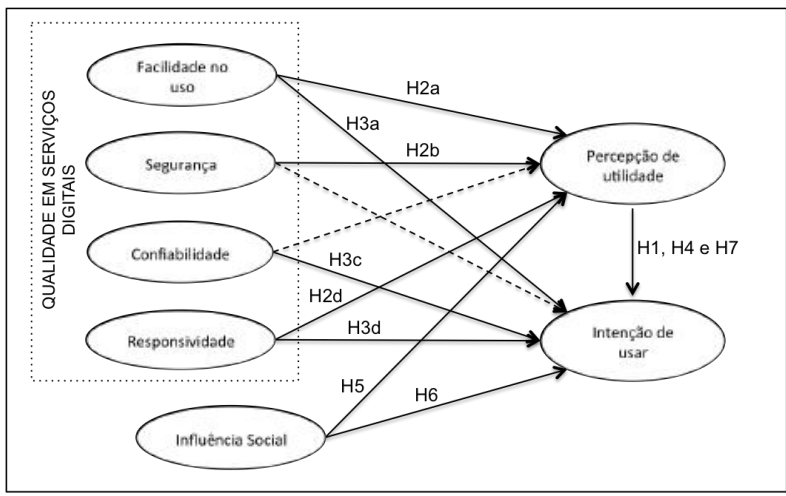

Figure 1: Relações de influência do modelo SaaSQual

\subsubsection{Percepção de utilidade - PU}

Percepção de utilidade é definido como o grau em que um indivíduo acredita que o uso de SaaS melhoraria o seu desempenho no trabalho. A maior diferença entre SaaS e software de uso geral reside na percepção de controle dos dados de negócio dos usuários [31]. A partir dos estudos pesquisados que utilizaram o modelo TAM, percebeu-se que há uma influência da percepção de utilidade sobre a intenção comportamental de usar.

Considerando a afirmativa anterior, é proposto avaliar a hipótese: H1 Percepção de utilidade tem um impacto positivo sobre a intenção de usar.

\subsubsection{Qualidade em serviços digitais (e-services)}

[7] questionam se a escala de qualidade aplicada ao contexto tradicional do mercado B2C (Bussines to Consumer) pode ser aplicada aos sistemas SaaS. Para [23], qualidade de serviço pode ser ilustrados em atributos como: tempo de resposta, confiabilidade e segurança de software. Quando clientes tem acesso a sistemas como serviço, questões como
Table 1: Construtos do modelo SaaSQual, extraído e adaptado de [7].

\begin{tabular}{|c|l|}
\hline Fator & Descrição \\
\hline \multirow{1}{*}{ FU } & $\begin{array}{l}\text { V01 - Acho fácil aprender a utilizar um sistema } \\
\text { XYZ }\end{array}$ \\
\cline { 2 - 3 } & $\begin{array}{l}\text { V02 - Acho fácil tornar-me habilidoso no uso de } \\
\text { um sistema XYZ }\end{array}$ \\
\cline { 2 - 3 } & $\begin{array}{l}\text { V03 - Nos sistemas XYZ que utilizo posso afir- } \\
\text { mar que conheço todas as suas funções }\end{array}$ \\
\hline \multirow{6}{*}{ SE } & $\begin{array}{l}\text { V04 - Meus dados particulares estão seguros } \\
\text { sendo guardados nos sistemas XYZ }\end{array}$ \\
\cline { 2 - 3 } & $\begin{array}{l}\text { V05 - Ninguém pode monitorar qualquer opera- } \\
\text { ção realizada por mim em sistemas XYZ }\end{array}$ \\
\cline { 2 - 3 } & $\begin{array}{l}\text { V06 - Ninguém pode ter acesso aos meus da- } \\
\text { dos particulares guardados no sistema XYZ, so- } \\
\text { mente se autorizado por mim }\end{array}$ \\
\hline & $\begin{array}{l}\text { V07 - Os sistemas XYZ sempre estão disponíveis } \\
\text { para o meu uso, e nunca estão fora do ar }\end{array}$ \\
\cline { 2 - 3 } & $\begin{array}{l}\text { V08 - Não ocorre problemas nos sistemas XYZ } \\
\text { durante o seu uso }\end{array}$ \\
\cline { 2 - 3 } & $\begin{array}{l}\text { V09 - Quando envio instruções para o sistema } \\
\text { XYZ, elas são executadas sem interrupção. }\end{array}$ \\
\hline \multirow{2}{*}{ RE } & $\begin{array}{l}\text { V10 - Para os usuários de sistemas XYZ, há vá- } \\
\text { rios canais de atendimento disponíveis que po- } \\
\text { dem ser utilizados para o suporte }\end{array}$ \\
\cline { 2 - 3 } & $\begin{array}{l}\text { V11 - O serviço de atendimento ao cliente dos } \\
\text { sistemas XYZ responde às minhas dúvidas cor- } \\
\text { retamente }\end{array}$ \\
\cline { 2 - 3 } & $\begin{array}{l}\text { V12 - O serviço de atendimento ao cliente dos } \\
\text { sistemas XYZ responde às minhas necessidades } \\
\text { no tempo esperado }\end{array}$ \\
\hline &
\end{tabular}

facilidade do uso (FU), segurança dos dados (SE), confiabilidade $(\mathrm{CO})$ de rede e serviços de atendimento ao usuário (responsividade - RE) são evidenciadas, e todas elas possuem impacto na percepção de utilidade.

Para [7], facilidade de uso é uma das principais características de influencia no processo de utilização de SaaS no nível individual (usuário). Para um ganho real proveniente da aplicação de SaaS em um contexto, é necessário que os usuários consigam aplicar o modelo no seu dia-a-dia de trabalho. Portanto, a facilidade de uso é um dos fatores cruciais para a intenção de usar (IU), e constitui um impacto indireto sobre a intenção de usar pelo fato de influenciar também a percepção de utilidade $[5,6,28,29]$.

Problema de segurança são os fatores mais citados para desencorajar o uso de SaaS [31]. Como os usuários têm que usar Internet para armazenar e acessar dados, eles tem que sacrificar, em certa medida, o controle sobre os seus dados, que são os principais ativos em suas operações comerciais. Consideramos confiabilidade SaaS como a medida em que o sistema de SaaS é executado corretamente quando os indivíduos estão usando. É menos provável que os usuários aceitem quedas inesperadas do servidor e de rede quando eles estão lidando com operações críticas de negócios. Entretanto, tal situação é um fator inibidor na intenção de utilizar SaaS [7].

Assim, é proposto avaliar as seguintes hipóteses: 
Table 2: Construtos complementares do modelo SaaSQual, extraído e adaptado de [7].

\begin{tabular}{|c|c|}
\hline Variável & Descrição do item \\
\hline \multirow[t]{4}{*}{ IS } & $\begin{array}{l}\text { V13 - Dentro do meu ambiente de trabalho, } \\
\text { as pessoas que são importantes para mim } \\
\text { pensam que devo adotar sistemas XYZ }\end{array}$ \\
\hline & $\begin{array}{l}\text { V14 - Pessoas de meu convívio, e que me in- } \\
\text { fluenciam socialmente, pensam que devo uti- } \\
\text { lizar sistemas XYZ }\end{array}$ \\
\hline & $\begin{array}{l}\text { V15 - A direção da empresa em que traba- } \\
\text { lho tem sido prestativa na adoção e uso de } \\
\text { sistemas XYZ }\end{array}$ \\
\hline & $\begin{array}{l}\text { V16 - De uma maneira geral, a empresa que } \\
\text { trabalho tem incentivado e apoiado o uso de } \\
\text { sistemas XYZ }\end{array}$ \\
\hline \multirow[t]{4}{*}{$\overline{\mathrm{PU}}$} & $\begin{array}{l}\text { V17 - Eu acho sistemas XYZ úteis no meu } \\
\text { trabalho }\end{array}$ \\
\hline & $\begin{array}{l}\text { V18 - A utilização de sistemas XYZ tem me } \\
\text { permitido executar minhas tarefas mais ra- } \\
\text { pidamente }\end{array}$ \\
\hline & $\begin{array}{l}\text { V19 A utilização de sistemas XYZ tem me } \\
\text { possibilitado uma melhor produtividade no } \\
\text { trabalho }\end{array}$ \\
\hline & $\begin{array}{l}\text { V20 - Se eu utilizar os sistemas XYZ, as mi- } \\
\text { nhas chances de ser promovido serão maiores. }\end{array}$ \\
\hline \multirow[t]{3}{*}{ IU } & $\begin{array}{l}\text { V21 - Eu tenho a intenção de utilizar siste- } \\
\text { mas XYZ nos próximos meses }\end{array}$ \\
\hline & $\begin{array}{l}\text { V22 - Eu digo coisas positivas para outras } \\
\text { pessoas a respeito dos sistemas XYZ }\end{array}$ \\
\hline & $\begin{array}{l}\text { V23 - Eu recomendo o uso dos sistemas XYZ } \\
\text { às pessoas que me pedem conselhos }\end{array}$ \\
\hline
\end{tabular}

H2 Qualidade em serviços digitais tem um impacto positivo direto na percepção de utilidade.

H3 Qualidade em serviços digitais tem um impacto positivo direto na intenção de usar.

H4 Qualidade em serviços digitais tem um impacto positivo indireto na intenção de usar, através da percepção de utilidade.

A hipótese $\mathrm{H} 2$ é parcialmente atendida, porque as quatro dimensões da qualidade em serviços digitais têm diferentes efeitos sobre a percepção de utilidade. De acordo com [7], apenas a relação entre confiabilidade e percepção de utilidade não é suportado, e uma provável explicação é que a percepção de utilidade é relacionada principalmente com módulos funcionais do SaaS propriamente dito, no entanto, a confiabilidade é relacionada com muitos fatores que imputam a operação de um sistema SaaS.

Os resultados do trabalho de [7] também suportam a hipótese H3 parcialmente. Apenas a relação entre segurança e intenção de usar não é suportada. Uma possível explicação, de acordo com o autor, é que diferentes experiências de utilização do SaaS têm um diversa percepção a respeito de segurança em SaaS.

\subsubsection{Influência social - IS}

A influência social é o grau em que um indivíduo percebe que outras pessoas importantes acreditam que ele ou ela deve usar o novo sistema [29]. Durante a adoção de SaaS, as pessoas podem interagir entre si, e também na comunidade, discutindo e influenciando sobre a utilização [7].

Assim, é proposta em sistema SaaS:

H5 Influência social tem um impacto positivo direto na percepção de utilidade.

H6 Influência social tem um impacto positivo direto na intenção de usar.

H7 Influência social tem um impacto positivo indireto na intenção de usar, através da percepção de utilidade.

\section{ANÁLISE DE DADOS}

Com o objetivo de reduzir o número de questões (baseado em uma escala do tipo Likert) a um grupo menor de variáveis que explicam de forma mais objetiva o grau de concordância com a adoção de sistemas na modalidade SaaS pelos funcionários de planos de saúde, uma análise fatorial pelo método de componentes principais foi utilizada.

Para avaliar a existência de conveniência do modelo da análise fatorial, o teste de esfericidade de Bartlett e a medida de adequacidade da amostra de Kaiser-Meyer-Olkin (KMO) foram utilizados [10].

Para determinar o número de fatores das 23 questões estudadas utilizam-se as técnicas de determinação com base em autovalores e na porcentagem de variância explicada. Considera-se como parâmetro de avaliação para a análise fatorial um percentual de variância explicada acumulada superior a $60 \%$. Primeiramente, utilizam-se o parâmetros referentes ao modelo SEM rotação (Modelo inicial), e, caso o modelo de análise fatorial não se ajuste adequadamente, utiliza-se a análise fatorial pelo método de componente principal com rotação do tipo VARIMAX com o objetivo de se obter um melhor ajuste para o modelo, bem como, determinar o número mínimo de fatores que respondam pela máxima variância nos dados pesquisados.[10]

Além disso, foram examinadas as diferenças entre as correlações observadas (matriz de correlação com todas as questões originais) e as correlações reproduzidas (correlações estimadas pela matriz de constructos), onde, essas diferenças chamadas de resíduos quando apresentam valores muito baixos indicam um bom ajuste do modelo gerado [10].

As medidas de comunalidade e as medidas de adequacidade da amostra para cada uma das questões participante da análise fatorial final também foram utilizadas.

Por fim, neste estudo foram apresentadas as medidas descritivas Mínimo, Máximo, Mediana, Média e desvio-padrão (d.p.), além, de percentuais como medidas para descrever os resultados das variáveis estudadas [14].

\subsection{Análise fatorial}

\subsubsection{Primeiro modelo (Modelo inicial)}

$\mathrm{Na}$ realização da análise fatorial foram utilizadas todas 23 questões estudadas no questionário que avalia o grau de concordância com a adoção de sistemas na modalidade SaaS, 
onde, os entrevistados foram solicitados a darem notas de 1 (discordo totalmente) a 7 (concordo totalmente) para as afirmativas propostas.

Na realização da análise fatorial inicial (primeiro modelo) foram utilizadas todas as questões que compõem o questionário que avalia o grau de concordância com a adoção de sistemas na modalidade SaaS. E, apesar do primeiro modelo mostrar-se conveniente na aplicação desta técnica estatística no que tange os resultados que avaliam a sua adequacidade, os auto-valores e o percentual de variância explicada e as medidas das cargas fatoriais foi necessária a retirada de uma das questões do questionário (questão $\mathrm{n}^{\circ} 21$ ), pois, além desta questão compartilhar com as demais questões uma baixa proporção de variância, a questão 21 quando retirada contribui com uma melhora no resultado para a confiabilidade interna do fator a que pertence esta questão, ou seja, o Alfa de Cronbach aumenta de 0,71, no geral, para 0,77 (este valor refere-se à Medida de Alfa de Cronbach quando a questão é retirada). Com isso, um novo modelo é executado após a retirada da questão não adequada e novos resultados são apresentados e reavaliados.

\subsubsection{Segundo modelo}

E, conforme mostram os resultados na avaliação do segundo modelo, existe a conveniência da aplicação desta técnica estatística no que diz respeito os resultados que avaliam a medida de adequacidade (KMO), o teste de esfericidade de Bartlett e as medidas dos auto-valores, do percentual de variância explicada e, também, das medidas das cargas fatoriais, entretanto, foi necessária a retirada de outras duas questões do questionário (questões 4 e 5). Pois, com as exclusões destas questões da análise fatorial, as medidas de consistência interna (valores das medidas do Alfa de Cronbach quando as questões 4 e 5 são retiradas) são significativamente aumentadas em comparação com as demais questões que compõem o fator latente correspondente. Ou seja, com a retirada da questão 4 do fator ?FU - Facilidade de uso? o Alfa de Cronbach aumenta para 0,86 (muito acima quando as questões 2 e 3 são retiradas deste fator) e com a retirada da questão 5 do fator ?SE - Segurança? o Alfa de Cronbach aumenta para 0,84 (muito acima quando as questões 6 e 7 são retiradas deste fator).

\subsubsection{Terceiro modelo (Modelo final)}

Com isso, um terceiro modelo (e final) foi gerado com as exclusões das questões 21,4 e 5 conforme discussão dos resultados dos dois modelos fatoriais iniciais (primeiro e segundo modelos). E, os resultados mostram que a terceira análise fatorial (análise final) é satisfatória, isto é, foi possível identificar construtos latentes que explicam as interdependências entre as 20 questões restantes do questionário que avalia o grau de concordância com a adoção de sistemas na modalidade SaaS. Portanto, as correlações entre o conjunto de variáveis (20 questões) de interesse são significativas e o processo de redução e sumarização de dados é viável.

O teste KMO apresentou uma medida igual a 0,75 , valor este muito superior ao valor de referência de 0,50 , portanto, indicando que a análise fatorial é adequada ao conjunto de questões pesquisado. Além disso, observa-se pelo teste de Esfericidade de Bartlett que existe um conjunto de correla- ções significativas $(\mathrm{p}<0,05)$ entre as 20 questões que compõem o questionário baseando-se nas respostas dadas pelos 147 funcionários de planos de saúde, ou seja, foi verificado um alto índice de interdependência / inter-relações entre as 20 questões finais estudadas nesta pesquisa. Assim, baseado nos pressupostos desta análise apresentados, a técnica estatística da análise fatorial pode ser aplicada para a redução e sumarização de variáveis com o intuito de identificar conjuntos de variáveis que formam dimensões latentes (fatores).

E com o objetivo de determinar o número de fatores latentes, no que se tange às 20 questões estudadas do questionário, foram utilizados parâmetros com base nas medidas dos autovalores e da porcentagem da variância total explicada gerado pela análise fatorial baseado no método de componentes principais. Com os resultados obtidos pelo método de componentes principais, foram identificados seis fatores latentes cujos autovalores foram superiores a um e com uma porcentagem de variância total explicada acumulada superior a $60 \%$ (valor de corte de referência para considerar uma análise adequada). Nesta pesquisa, os seis fatores gerados explicam $76,9 \%$ de toda a variância do conjunto das 20 questões que avalia o grau de concordância com a adoção de sistemas na modalidade SaaS, sendo ainda utilizada a análise de componente principal com rotação Varimax com o intuito de se obter um melhor ajuste para o modelo ao buscar uma solução mais simples e pragmaticamente mais significativa, bem como, determinar o número mínimo de fatores que respondam pela máxima variância explicada nos dados do questionário pesquisado. Portanto, com estes resultados trabalhar-se-á com seis fatores independentes que subdivididos em blocos de questões explicam o grau de concordância dos entrevistados com a adoção de sistemas na modalidade SaaS. Com isso, conceitos teóricos são criados para cada um dos fatores latentes gerados pela análise fatorial, contribuindo então para uma redução dos dados através do cálculo dos escores de cada Fator latente e, então, substituindo as variáveis originais pesquisadas.

Resumindo, apesar de existirem vários fatores correlacionados, seus níveis de correlações encontrados são considerados fracos confirmando a interdependência entre os seis fatores produzidos pela análise fatorial. E, para finalizar, cada fator é calculado através de uma combinação linear das questões indicadas de acordo com as cargas fatoriais significativas e dividindo-se pelo número de questões que o compõe.

O coeficiente Alfa de Cronbach foi utilizado para avaliar a confiabilidade da consistência interna das questões que compõem cada um dos seis fatores gerados pela análise fatorial. Os seis fatores estudados apresentaram medidas de Alfa de Cronbach muito superiores a 0,60 , indicando confiabilidade satisfatória de consistência interna. Cabe aqui destacar que no questionário original o pesquisador idealizou a ocorrência de sete fatores que continham, a princípio, de três a quatro questões por fator. Como este estudo tem um objetivo de identificar, em funcionários de planos de saúde, no Brasil, a composição destas inter-relações entre as 23 variáveis originais, dado a natureza da técnica estatística utilizada, novos reagrupamentos eram esperados, ou seja, os fatores seriam compostos por agrupamentos de questões que não acompanhassem o modelo original de sete fatores, além da perda de algumas variáveis por não se correlacionarem ou explicarem 
os novos fatores gerados.

A tabela 3 demonstra a descrição dos seis fatores gerados pela análise fatorial, no geral.

Table 3: Medidas descritivas das medidas dos seis fatores gerados pela análise fatorial

\begin{tabular}{c|ccccc}
\hline fatores & \multicolumn{5}{|c}{ Medidas descritivas } \\
\cline { 2 - 6 } & Mínimo & Máximo & Mediana & Média & d.p. \\
\hline PU & 3,2 & 7,0 & 5,8 & 5,7 & 0,9 \\
IS & 1,0 & 7,0 & 5,0 & 4,9 & 1,2 \\
RE & 1,0 & 7,0 & 4,7 & 4,5 & 1,1 \\
CO & 1,0 & 6,7 & 4,7 & 4,3 & 1,4 \\
FU & 1,0 & 7,0 & 6,0 & 5,8 & 0,9 \\
SE & 1,0 & 7,0 & 3,0 & 3,4 & 1,6 \\
\hline
\end{tabular}

\subsection{Avaliação de consistência interna do mo- delo original}

Ressalta-se que foram observadas correlações significativas $(\mathrm{p}<0,05)$ entre quase todos os fatores originais de referência, porém, os valores dos coeficientes de correlação apresentam valores considerados fracos ou muito fracos, ou seja, valores abaixo ou próximos a 0,40. As exceções são as correlações entre os fatores ?IS - Influência social? e ?PU Percepção de utilidade? e entre os fatores ?PU - Percepção de utilidade? e ?IU - Intenção de usar? com coeficientes de correção variando de 0,53 a 0,60 , respectivamente, entretanto, considerados estes coeficientes como variando de fracos a moderados $(0,40<\mathrm{r}<0,70)$.

\section{RESULTADOS}

A metodologia de análise de conglomerados (cluster) baseado no método K-médias foi utilizada com o objetivo de determinar perfis diferenciados quanto aos fatores gerados pela análise fatorial de acordo com o questionário (23 questões).

Com o objetivo de comparar os dois grupos independentes quanto à medida de uma variável de interesse do tipo escalar é utilizado o teste $t$ de Student para amostras independentes. Trata-se de um teste paramétrico que tem como objetivo comparar médias entre dois grupos distintos de interesse. Isto é, este teste avalia se existe diferença significativa ou não entre os dois grupos quanto às médias das medidas em cada uma das variáveis de interesse [13].

\subsection{Caracterização dos respondentes}

Pôde ser observado que o universo masculino foi predominante no conjunto de respondentes, porém este viés pouco interferiu nos resultados.

O resultado obtido demonstra que as ferramentas de mensagens eletrônicas (e-mail) são as mais utilizadas no formato SaaS, porém é importante destacar que as ferramentas de suíte de escritório possuem representatividade nos resultados. O sistema menos utilizados são os sistemas de gestão de rotina, que são sistemas mais especialistas.

Sobre a distribuição dos níveis hierárquicos, a pesquisa conseguiu abranger uma boa distribuição entre os níveis de alta gerência (presidente e diretores), média gerência (gerentes,

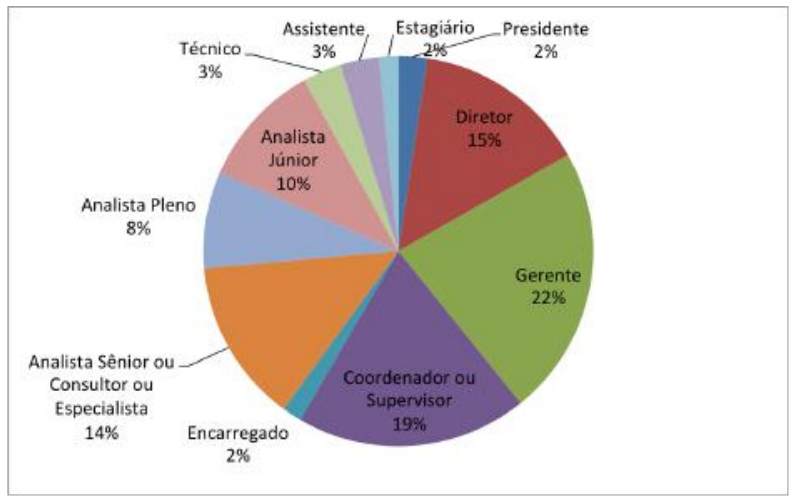

Figure 2: Distribuição do nível hierárquico dos respondentes

coordenadores e supervisores) e nível operacional (analistas, consultores, encarregados, técnicos, assistentes e estagiários).

\subsection{Análise de conglomerados}

Baseando-se nos seis fatores criados pela análise fatorial, técnica para redução e sumarização de dados usados na presente pesquisa, a análise de conglomerados (clusters) foi utilizada para identificar possíveis grupos de entrevistados, funcionários de empresas de planos de saúde, com características únicas. Os resultados indicaram a presença de dois conglomerados (grupos) distintos de respondentes baseados conjuntamente nos seis fatores, onde, $47,6 \%$ dos entrevistados apresentaram grau II (?alta concordância?) e 52,4\% apresentaram grau I (?baixa concordância?) de concordância com a adoção de sistemas na modalidade SaaS.

A tabela 4 apresenta os escores médios dos dois conglomerados (grupos) gerados por cada um dos seis fatores estudados, no geral. Além disso, estes resultados mostram que os dois grupos de respondentes formados diferem estatisticamente entre si, com exceção do Fator ?FU - Facilidade de uso?, ou seja, as médias de cada um dos demais cinco fatores avaliados diferem significativamente $(\mathrm{p}<0,05)$ entre os grupos com grau de concordância I e II. Portanto, cada grupo formado é único e caracterizado por homogeneidade interna em cada grupo. A figura 3 apresenta as médias dos seis fatores para cada um dos dois grupos que avaliam o grau de concordância dos entrevistados com a adoção de sistemas na modalidade SaaS, onde, em todos os fatores, com exceção do Fator ?FU - Facilidade de uso?, a média do grupo com Grau II de concordância é estatisticamente superior ao grupo de entrevistados com Grau I.

\section{CONCLUSÃO}

Os resultados apresentados nesta pesquisa corroboraram com os dados anteriormente encontrados na revisão da literatura. Para as operadoras de planos de saúde, o fator de maior concordância na adoção de sistemas na modalidade SaaS foi a facilidade de uso, seguido da percepção de utilidade. Nos dois casos, a média de aceitação foi de 5,9 e 5,7, respectivamente, em uma escala que vai até 7,0 . Em contrapartida, o fator segurança, seguido do fator confiabilidade, 
Table 4: Média dos seis fatores gerados pela análise fatorial em relação aos dois grupos

\begin{tabular}{l|cc|c|c}
\hline fatores & Grau I & Grau II & $\mathbf{p}$ & Conclusão \\
\hline PU & 5,5 & 5,9 & 0,052 & I $<$ II \\
IS & 4,5 & 5,3 & $<0,001$ & I $<$ II \\
RE & 4,1 & 5,0 & $<0,001$ & I $<$ II \\
CO & 3,5 & 5,2 & $<0,001$ & I $<$ II \\
FU & 5,7 & 5,9 & 0,159 & I $=$ II \\
SE & 2,2 & 4,6 & $<0,001$ & I $<$ II \\
\hline
\end{tabular}

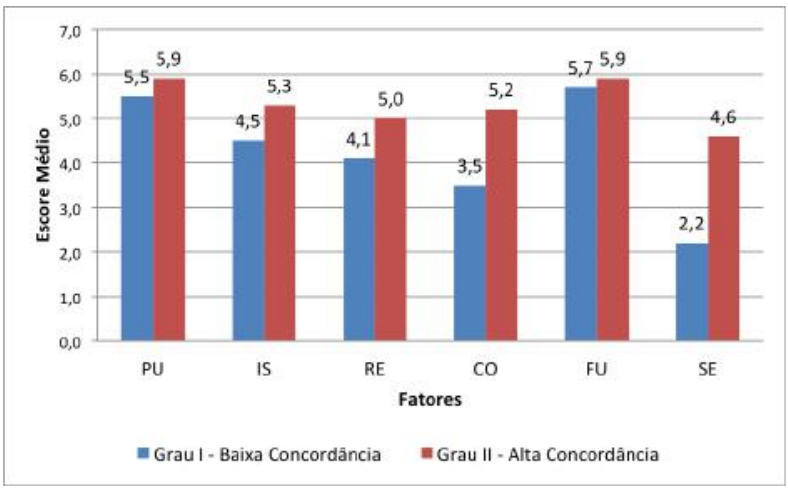

Figure 3: Média dos seis fatores gerados pela análise fatorial em relação aos dois grupos (clusters) de entrevistados formados pela análise de conglomerados

foram os itens de menor concordância, com valores de 3,4 e 4,3 respectivamente. Notou-se também que o aspecto sexo possui pouca influência na pesquisa. Apesar do número de respondentes do sexo masculino ser quatro vezes maior do que os respondentes do sexo feminino, os resultados para ambos apresentaram números semelhantes.

Conforme pôde ter sido averiguado na literatura, o principal fator motivador está relacionado à percepção de utilidade do sistema, enquanto o maior fator inibidor foi a qualidade do serviço disponibilizado. Ainda dentro do conceito de qualidade, um aspecto de destaque de inibição de uso dos sistemas na modalidade SaaS foi a segurança.

Por fim, foi percebido que o nível hierárquico dos respondentes influenciou no nível de aceitação. Enquanto que os níveis mais altos são mais conservadores no uso da tecnologia SaaS, os níveis mais baixos (Analista Júnior) possuem um alto nível de concordância na sua adoção.

\section{REFERENCES}

[1] ANS. Caderno de Informação da Saúde Suplementar: beneficiários, operadoras e planos. Technical report, Ministério da Saúde, Rio de Janeiro, 2013.

[2] E. Babbie. Métodos de pesquisas de survey. Editora UFMG, Belo Horizonte, 2005.

[3] E. Bublitz. Catching The Cloud. National Underwriter $P C$, pages $12-16,2010$.

[4] J. a. J. S. da FONSECA. Metodologia da pesquisa científica. Curso de Especialização em Comunidades Virtuais de Aprendizagem - Informática Educativa. Universidade Estadual do Ceará, 2002.
[5] F. D. Davis. A technology acceptance model for empirically testing new end-user information systems: Theory and results. $\mathrm{PhD}$ thesis, Massachusetts Institute of Technology, 1986.

[6] F. D. Davis, R. P. Bagozzi, and P. R. Warshaw. User Acceptance of Computer Technology: A Comparison of Two Theoretical Models. Management Science, 35(8):982-1003, Aug. 1989.

[7] J. Du, J. Lu, X. Wu, and J. Li. Research on the influential factors of SaaS user acceptance. pages 182-185, Nov. 2012.

[8] H. Freitas, M. Oliveira, A. Z. Saccol, and J. Moscarola. O Método de Pesquisa Survey. RAUSP (Revista de Administração da Universidade de São Paulo), 35:105-112, 2000.

[9] W. Gu and Y. Liu. SaaS-Based Services Information Technology Acceptance Model. volume 1, pages 87-90, Sept. 2011.

[10] J. Hair, W. Black, B. Babin, R. Anderson, and R. Tatham. Análise multivariada de dados. Bookman, 2009.

[11] J. Hair Jr, B. BABIN, A. MONEY, and P. SAMOUEL. Fundamentos de métodos de pesquisa em administração. Porto Alegre: Bookman, 2005.

[12] M. J. Harnisch, T. Puchleitner, M. Reinisch, and I. Uitz. Model of a Personalization-Based Agent System for Early Product Adoption Phases. pages 3457-3466, Jan. 2013.

[13] R. Johnson and G. Bhattacharyya. Statistics: Principles and methods. 1996.

[14] R. Johnson and D. Wichern. Applied multivariate statistical analysis, volume 4. Prentice hall Englewood Cliffs, NJ, 1992.

[15] W. Kim. Cloud Computing : Today and Tomorrow. Journal of Object Technology, 8:65-72, 2009.

[16] J. D. Lasica. Identity in the Age of Cloud Computing: The next-generation Internet's impact on business, governance and social interaction. 2009.

[17] Y.-C. Lee, M.-L. Li, T.-M. Yen, and T.-H. Huang. Analysis of adopting an integrated decision making trial and evaluation laboratory on a technology acceptance model. Expert Systems with Applications, 37(2):1745-1754, 2010.

[18] J. Lewandowski, A. Salako, and A. Garcia-Perez. SaaS Enterprise Resource Planning Systems: Challenges of Their Adoption in SMEs. pages 56-61, Sept. 2013.

[19] D. Malta and A. Jorge. Breve caracterização da saúde suplementar. In ...da Saúde/Agência Nacional de Saúde Suplementar, pages 37-60. Rio de Janeiro, 2005.

[20] S. Marston, Z. Li, S. Bandyopadhyay, J. Zhang, and A. Ghalsasi. Cloud computing - The business perspective. Decision Support Systems, 51(1):176-189, Apr. 2011.

[21] P. Mell and T. Grance. The NIST Definition of Cloud Computing-Recommendations of the National Institute of Standards and Technology. NIST. NIST Special Publication, 2011.

[22] P. Miguel. Metodologia de pesquisa em engenharia de produção e gestão de operações. Elsevier, 2012.

[23] D. C. Novak. Internet access and capacity planning: Quantifying relationships between usage, capacity, and 
blocking. Telecommunications Policy, 34(5-6):309-322, June 2010.

[24] K. Potter and M. Smith. IT Metrics: IT Spending and Staffing Report 2011. G00210146, ..., (January), 2011.

[25] P. Roehrig. New Market Pressures Will Drive Next-Generation IT Services Outsourcing. Global Services, 2008.

[26] V. Venkatesh and H. Bala. Technology acceptance model 3 and a research agenda on interventions. Decision sciences, 39(2):273-315, 2008.

[27] V. Venkatesh and F. Davis. A model of the antecedents of perceived ease of use: Development and test*. Decision sciences, 27(3):451-481, 1996.

[28] V. Venkatesh and F. D. Davis. A Theoretical Extension of the Technology Acceptance Model: Four Longitudinal Field Studies. Management Science, 46(2):186, Feb. 2000.

[29] V. Venkatesh, M. G. Morris, G. B. Davis, and F. D. Davis. User acceptance of information technology: Toward a unified view. MIS quarterly, 27(3), 2003.

[30] T. Wang, L.-B. Oh, K. Wang, and Y. Yuan. User adoption and purchasing intention after free trial: an empirical study of mobile newspapers. Information Systems and e-Business Management, 11(2):189-210, June 2013.

[31] W.-W. Wu. Developing an explorative model for SaaS adoption. Expert Systems with Applications, 38(12):15057-15064, Nov. 2011.

[32] W.-W. Wu. Mining significant factors affecting the adoption of SaaS using the rough set approach. Journal of Systems and Software, 84(3):435-441, Mar. 2011. 